\author{
Aleksander Grm \\ E-mail: aleksander.grm@fpp.uni-lj.si \\ University of Ljubljana, Faculty of Martitime Technology and Transport, \\ Pot pomorščakov 4, 6320 Portorož, Slovenia
}

\title{
Analysis of Generic IGBEM for Lifting Hydrofoils
}

\begin{abstract}
Today the most crucial aspect in the preliminary vessel design stage is to make it as green/blue as possible. One of the exciting goals is the minimisation of vessel resistance. The use of hydrofoils to reduce the vessel draught and consequently, reduction in the vessel resistance is today one of the hottest design topics, especially for catamaran passenger vessels. In the present work, we discuss the issues related to the implementation of Isogeometric Analysis (IGA) Boundary Element Method (BEM) for the calculation of the hydrodynamic properties of lifting hydrofoils. The use of IGBEM allows numerical calculation of foil hydrodynamic properties without the traditional step of mesh generation using the CAD geometry directly. The analysis relies on the NURBS basis function with the generic Galerkin approach allowing identical solutions procedures for 2D or 3D problems. Method accuracy and computational times for a different number of Degrees of Freedom (DOF) in 2D are investigated.
\end{abstract}

Keywords: IGA, BEM, NURBS, lifting hydrofoil, numerical analysis

\section{Introduction}

Today, the main focus in primary research in the shipbuilding industry is on the improvement of green/blue ship performance. Ship performance depends on the hydrodynamic properties of the hull geometry and its keel, rudder, hydrofoil and other appendages. Hull and appendages also interact with free-surface that introduce additional nonlinear effect into flow properties. Interaction of free-surface flows with lifting surfaces constitutes a new complex problem, finding applications in the design of yachts and sailing boats and the performance of stabilisers, hydrofoils and similar devices [1-3]namely the so-called Neumann-Kelvin problem, following the formulation by Brard (1972.

Numerical methods to treat such complex systems face significant difficulties due to the simultaneous presence of many flow phenomena interacting in a highly nonlinear 
way. Not that the mathematical models are challenging to solve numerically, but also the complexity of the solution of such problems faces the barrier in computational time $[4,5]$.

Some of the ship flow phenomena can be simplified. In our case, we study the effect of lifting foils in foil assisted vessel geometry improving the hydrodynamic properties of the vessel [3].

\section{Mathematical model}

Modelling of fluid flow with free-surface in a context to resolve most of the flow phenomena is a very challenging task not only in modelling view but also in the computational perspective. For some family of problems, it is possible to reduce the complexity of models. Under certain flow conditions, Navier-Stokes equations simplify to Laplace equation for a potential field.

Neglecting the viscosity effect in Navier-Stokes equation, we are left with an inviscid family of problems, and viscous effects as drag, turbulence and boundary layer cannot be detected anymore. The study of the lift problem is part of the inviscid family of problems. Moreover, it is also assumed that the fluid motion is irrotational in our case. Based on these assumptions, it is possible to reduce the fluid flow description to the Laplace equation. One of the solutions of the Laplace equation is Boundary Integral Method.

Boundary integral method (BIM) solves the Laplace equation only on the boundary of the domain. This step reduces the computation of fluid flow only to the solution of fluid flow on the domain boundary. In our case, the boundary geometry is described with the NURBS [6] representation. Using NURBS as geometry representation, it is possible to use the NURBS function as the basis functions for the numerical solution of BIM problems implemented in BEM $[7,8]$. Formulation of the original BIM problem for the solution of the Laplace equation is done in a variational formulation known as Galerkin problem [8]. In the end, the boundary integral equation (BIE) has to be solved. In this case, the general approach to the solution of the BIE is considered. The general approach treats the problem of singular integral as it is using the special integral techniques to solve singular integrals [9-11].

In Figure 1, the notation of the domain, the boundary orientation, normal and tangent vector are shown for a $2 \mathrm{D}$ case. 


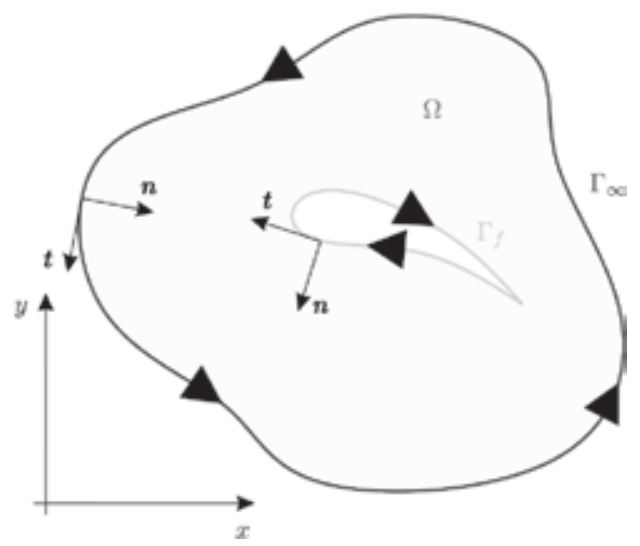

Figure 1: Computational domain $\Omega$ with the inner boundary $\Gamma_{f}$ and the external boundary $\Gamma_{\infty}$ in $2 D$.

The problem to be solved is the following

$\nabla^{2} u=0$, in $\Omega$

$\nabla u \cdot \boldsymbol{n}=-\boldsymbol{v}_{\infty} \cdot \boldsymbol{n}$, on $\Gamma_{f}$

$\lim _{\boldsymbol{x} \rightarrow \infty} u(\boldsymbol{x})=0$,

$\lim _{x \rightarrow \infty} \frac{\partial u}{\partial t}(x)=0$ (time change),

$\lim _{\boldsymbol{x} \rightarrow \infty} p(\boldsymbol{x})=p_{a}$ (atmospheric pressure),

where are $\boldsymbol{v}, \boldsymbol{n}, \boldsymbol{x} \in R^{n}$, with $\mathrm{n}=\{2,3\}$, and $\mathrm{u}=\mathrm{u}(\mathrm{x}, \mathrm{t})$ is the potential perturbation. Solution $u$ defines the velocity vector field

$v=\nabla \Phi$,

with potential $\Phi=\phi_{\infty}+u=\boldsymbol{v}_{\infty} \cdot \boldsymbol{x}+u$, and velocity $\lim _{x \rightarrow \infty} \boldsymbol{v}=\boldsymbol{v}_{\infty}$.

By the linearity of the Laplace operator, the solution of the velocity vector field $\mathrm{v}$ is obtained only by solving the problem .

On the inner boundary $\Gamma_{f}$ the homogeneous Neumann non-penetration boundary condition must be imposed

$\nabla \Phi \cdot \boldsymbol{n}=0 \rightarrow \nabla u \cdot \boldsymbol{n}+\boldsymbol{v}_{\infty} \cdot \boldsymbol{n}=0$

$\nabla u \cdot \boldsymbol{n}=-\boldsymbol{v}_{\infty} \cdot \boldsymbol{n}$

The problem can be reformulated into the integral form. In the end, the BIE problem must be solved 


$$
\frac{1}{2} u(\boldsymbol{x})=\int_{\Gamma}^{P V} G \nabla u \cdot \boldsymbol{n} d s-\int_{\Gamma} u \nabla G \cdot \boldsymbol{n} d s, \boldsymbol{x} \in \Gamma_{f},
$$

where $\mathrm{G}$ is the Green function and PV is the principal value integral [11].

Using NURBS representation, spatial coordinates $\boldsymbol{x}$, potential perturbation $\mathrm{u}$, normal and tangent vector $\boldsymbol{n}, \boldsymbol{t}$ and other variables can be converted into the form using only arc-length parameter $\mathrm{t}$ as the independent variable (in the $3 \mathrm{D}$ situation we would need two parameters). The Equation can be now converted to

$$
\begin{aligned}
\frac{1}{2} u(t) & +\int_{0}^{1} u(\tau) \nabla G(t, \tau) \cdot \boldsymbol{n}(\tau) J(\tau) d \tau= \\
& -\int_{0}^{1} G(t, \tau)\left(\boldsymbol{v}_{\infty} \cdot \boldsymbol{n}(\tau)\right) J(\tau) d \tau, \quad t \in[0,1] .
\end{aligned}
$$

This is Fredholm integral equation of the $2^{\text {nd }}$ kind for the unknown function $u(x)$, where $x \in \Omega$.

In our case, the Eq. is solved in a variational way. For example, the other possibility is to solve it in a colocation way [8] a new methodology for solving partial differential equations (PDEs. Let us rewrite the Eq. in a form to seek the unknown function $\mathrm{u}(\mathrm{t})$

$$
\frac{1}{2} u(t)+(P u)(t)=(Q)(t),
$$

where

$$
\begin{aligned}
& (P u)(t):=\int_{0}^{1} u(\tau) \nabla G(t, \tau) \cdot \boldsymbol{n}(\tau) J(\tau) d \tau, \\
& (Q)(t):=-\int_{0}^{1} G(t, \tau)\left(\boldsymbol{v}_{\infty} \cdot \boldsymbol{n}(\tau)\right) J(\tau) d \tau .
\end{aligned}
$$

Now the problem can be written into the variational form. Unknown function $u \in U$ and test function $v \in V$, can be from the same set of functions, so $U=V$. Eq. is multiplied by $\mathrm{v}$ and integrated such that we obtain the following variational problem:

Find $u \in V$ such that

$$
a(u, v)=q(v), \forall v \in V,
$$

where

$$
a(u, v):=m(u, v)+p(u, v) .
$$


The bilinear in linear forms are

$$
\begin{aligned}
& m(u, v):=\frac{1}{2} \int_{0}^{1} u(t) v(t) d t, \\
& p(u, v):=\int_{0}^{1}(P u)(t) v(t) d t, \\
& q(v):=\int_{0}^{1}(Q)(t) v(t) d t .
\end{aligned}
$$

Problem is written in the general variational form in an arbitrary spatial dimension. To solve it in a 2D the following Green functions are used

$$
\begin{aligned}
& G(t, \tau):=-\frac{1}{2 \pi} \ln \|\boldsymbol{r}(t)-\boldsymbol{r}(\tau)\|, \\
& \nabla G(t, \tau):=\frac{1}{2 \pi} \frac{\boldsymbol{r}(t)-\boldsymbol{r}(\tau)}{\|\boldsymbol{r}(t)-\boldsymbol{r}(\tau)\|^{2}},
\end{aligned}
$$

where $\boldsymbol{r}(t)$ is the position vector on the inner boundary $\Gamma_{f}$ written in NURBS representation.

\section{Results}

Implementation of the problem (7) is done in Matlab. Assembling of the system (7) is very slow if written in Matlab, because of many for loops. For this purpose, we add custom Matlab functions written in $\mathrm{C}++$. Integrals are solved with Gauss-Legendre quadrature rule [12]. However, singular integrals were not solved as PV integrals but were solved with a self-adaptive procedure using Telles map [13]. As it will be observed this approach is not accurate enough when we compute lifting type of faces because the final results depend on the gradient of the solution. The improvement of the accuracy of the results is made by the use of special techniques, like in [14], for the computation of singular integrals.

The test benchmark problem is the solution of flow around the infinite cylinder (flow around the circle in 2D). The shape of the circle geometry is the NURBS curve of degree $p=2$

$$
\begin{aligned}
& U=\{0,0,0,1 / 4,1 / 4,1 / 2,1 / 2,3 / 4,3 / 4,1,1,1\}-\text { knots, } \\
& W=\{1,1 / \sqrt{2}, 1,1 / \sqrt{2}, 1,1 / \sqrt{2}, 1,1 / \sqrt{2}, 1\} \text { - weights, } \\
& C P_{x}=\{1,1,0,-1,-1,-1,0,1,1\}-\text { control points } x \text { coordinate, } \\
& C P_{y}=\{0,-1,-1,-1,0,1,1,1,0\} \text { - control points y coordinate, }
\end{aligned}
$$


describing it exactly. The solution of potential perturbation $u$ was obtained for different NURBS degree $p$ and compared to the analytical one [11]. The relative error $\epsilon$ with respect to the exact solution is shown in Figure 2. The method shows very good convergence results. Computational timings with respect to DoF are shown in Figure 3.

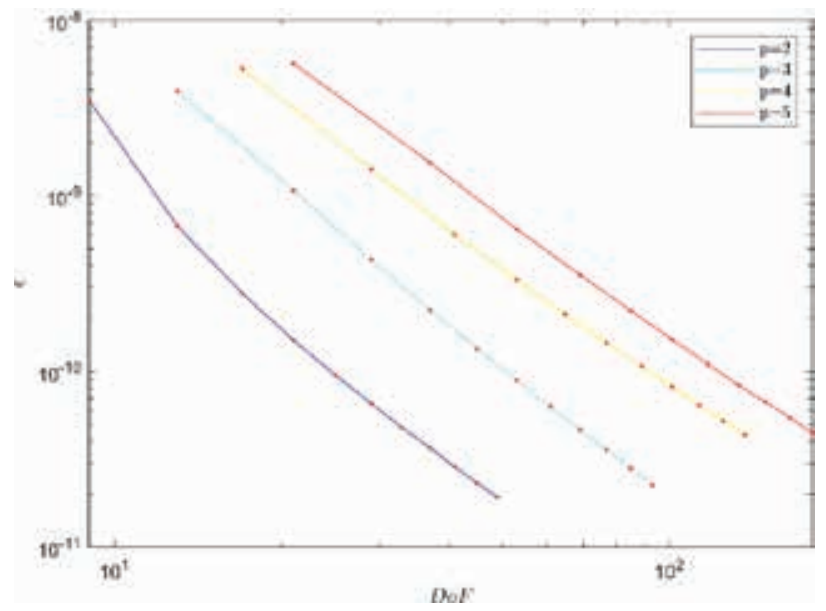

Figure 2: Method convergence for p refined solution for circle geometry.

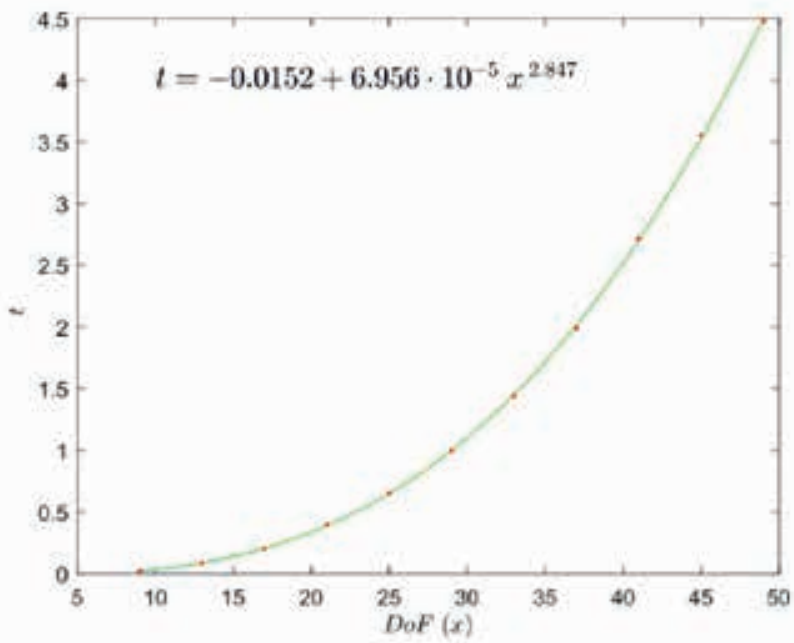

Figure 3: Computational times with respect to DoF ( $t$ is in seconds)

The variational approach shows good convergence properties on the benchmark problem, but the computational times are increased almost by the power of 3 . The implementation of the method is in pure sequential mode. If the implementation would 
be parallelised, it would probably reduce the computational times for an order or more. This is one of the future tasks.

Next, the method was tested for two NACA profiles, NACA0012 and NACA4412. To solve the lifting type of faces, as NACA profiles are, it was needed to pose additional constraints in the problem . The constraints are known as Kutta condition $[15,16]$. The same method is used to solve mentioned NACA profiles as was used for the benchmark problem with the extension of Kutta condition.

NURBS functions are very open and its use in BEM as basis functions for the problem it needs functions to be at least of degree $p \geq 3$. There are two ways to interpolate NACA profiles with NURBS function: use low order functions with more control points or use higher-order functions with fewer control points. Both versions were tested revelling that the higher-order function behaves much better than the low order one, but none of them gives accurate results yet. The reason behind is low accuracy of the numerical integration method that was used to evaluate the singular integrals. DoF for the interpolant of the NURBS curve is smaller for high order degree functions compared to low order one to achieve the same interpolation error.

Results of the pressure distribution for the NACA profiles are compared to the results from the XFoil program [17].

$$
C p=1-\frac{|\nabla \Phi|^{2}}{\left|\boldsymbol{v}_{\infty}\right|^{2}}
$$

Different tests were done to test the numerical method for different NURBS interpolants degree, from $p=3$ to $p=9$ with different number of DoF. Best results were obtained for NURBS degree $p=6$ and $\mathrm{DoF}=10$. The interpolated profiles for NACA0012 and NACA4412 are shown in Figure 4. Achieved interpolation accuracy for both cases was almost identical. Calculation of pressure distribution $C p$ for the profile b) are shown in Figure 5. The results are far from the expected results, like the one obtained with the XFoil program.

The best results are for the case for the angle of attack $\alpha=0^{\circ}$. Increasing the angle of attack the error in results is rising. $\mathrm{Cp}$ is calculated from the gradient of the potential (Eq. ). A small change in the potential $\Phi$ reflects a significant change in its gradient $\nabla \Phi$, . If the accuracy of the numerical evaluation of singular integrals is to low, it can reflect as a problem observed in Figure 5. 


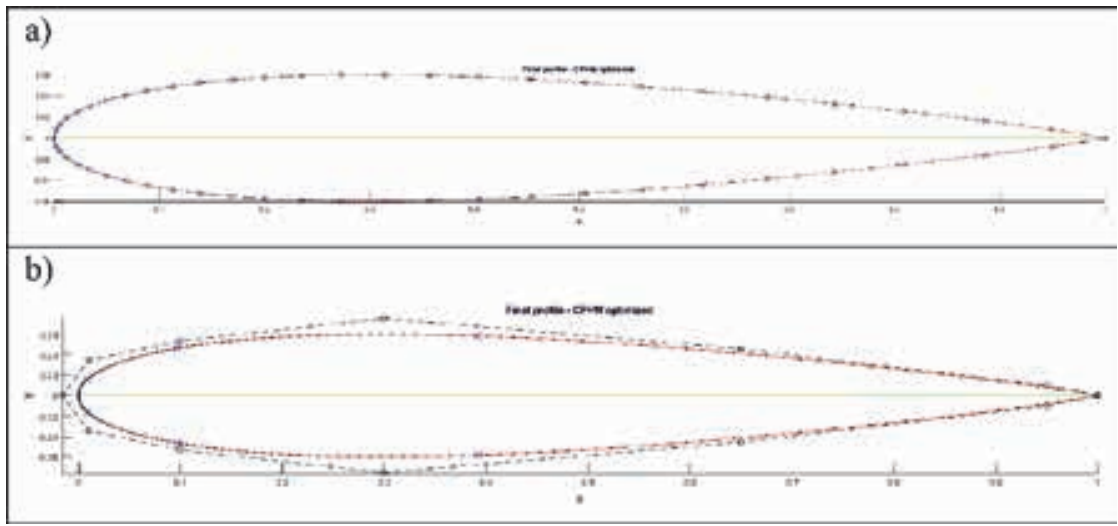

Figure 4: NURBS interpolants for NACA0012 profile, circles are control points, crosses are knots positions; a) $p=3, N=51$; b) $p=6, N=11$.

a)

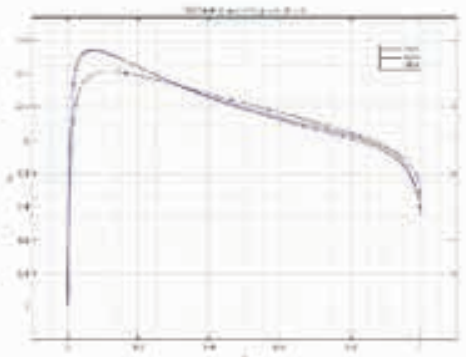

c)

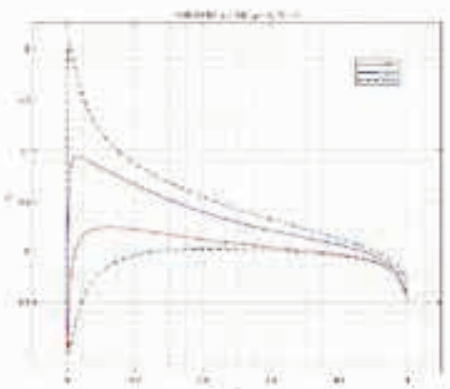

b)

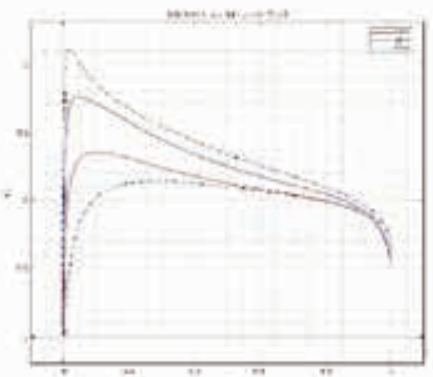

d)

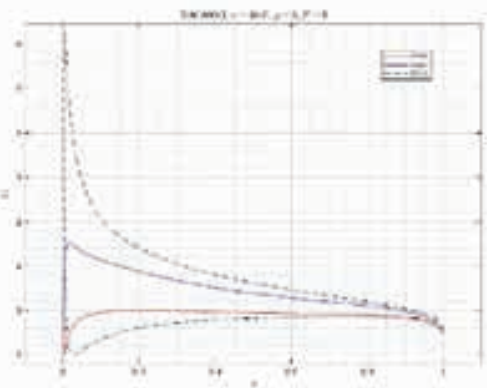

Figure 5: Pressure distribution Cp for NACA0012 profile; red - lower zone, blueupper zone, dashed -Xfoil; a) $\alpha=0^{\circ}$, b) $\alpha=3^{\circ}$, c) $\alpha=5^{\circ}$, d) $\alpha=10^{\circ}$

For the second test, the non-symmetric profile NACA4412 shown in Figure 6 was chosen. Results for the pressure distribution are shown in Figure 7. In the case of the non-symmetric profile, results show only the initial correct trend. The rest of the profile, especially the solution at the profile tail is entirely wrong. 


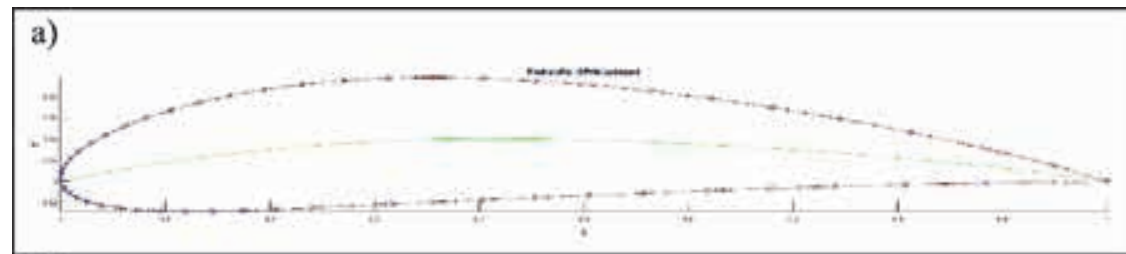

b)

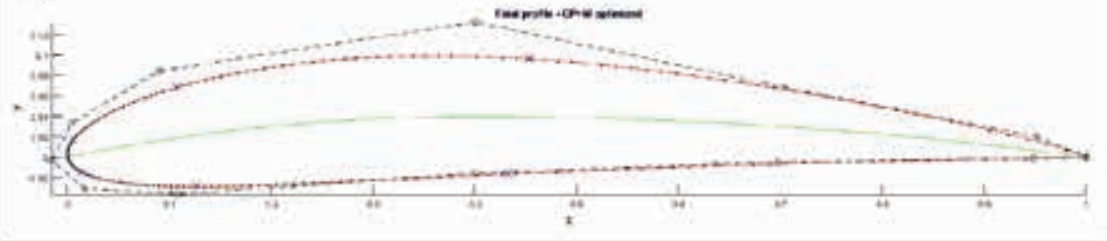

Figure 6: NURBS interpolants for NACA4412 profile, circles are control points, crosses are knots positions; a) $p=3, N=51$; b) $p=6, N=11$.

a)

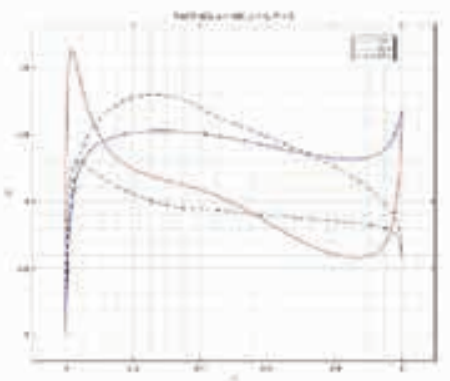

c)

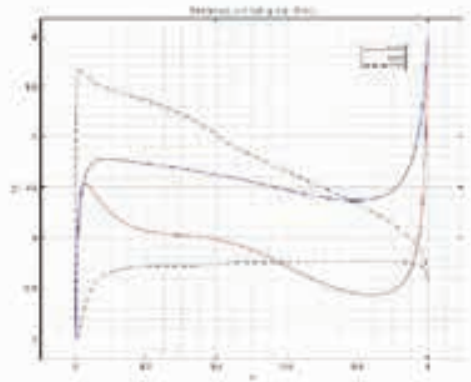

b)

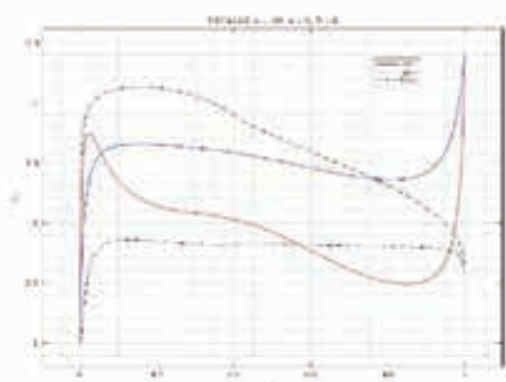

d)

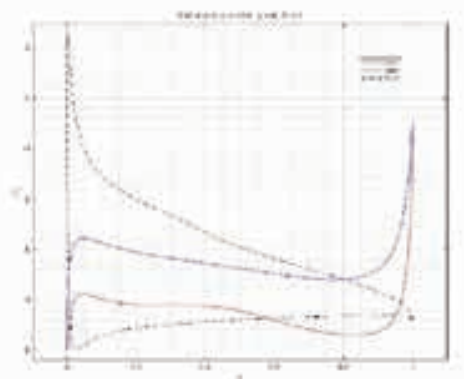

Figure 7: Pressure distribution Cp for NACA0012 profile; red-lower zone, blue upper zone, dashed-Xfoil; a) $\alpha=0^{\circ}$, b) $\alpha=3^{\circ}$, c) $\alpha=5^{\circ}$, d) $\alpha=10^{\circ}$ 


\section{Discussion}

In the present work, the general IGBEM for the calculation of pressure distribution for lifting profiles based on NURBS functions is presented. The power of the method is in the exact representation of foil geometry. At this stage, it still lacks the accuracy in the numerical evaluation of singular integrals.

The method was tested in two different tests with two NACA profiles and observed that for the symmetric profiles with high NURBS degree functions and low number of DoF will produce an incorrect but still informative result, even if the numerical evaluation of singular integrals is not accurate enough. In the case of non-symmetric profiles at the moment, the method completely fails. The reason in the incorrect results is in the accuracy of the numerical integration method for the evaluation of singular integrals. Even the use of self-adaptive mapping approach proposed by Telles [13] does not help in the accuracy of the evaluation of singular integrals. New approaches are needed and are tested to improve the calculation of potential gradient.

With the stable method, it is possible to move towards the interaction of free surface and 3D case.

\section{References}

1. Ginnis AI, Kostas K V., Politis CG, Kaklis PD, Belibassakis KA, Gerostathis TP, et al. Isogeometric boundary-element analysis for the wave-resistance problem using T-splines. Comput Methods Appl Mech Eng 2014;279:425-39. doi:10.1016/j.cma.2014.07.001.

2. Belibassakis KA, Gerostathis TP, Kostas K V., Politis CG, Kaklis PD, Ginnis AI, et al. A BEM-isogeometric method for the ship wave-resistance problem. Ocean Eng 2013;60:53-67. doi:10.1016/j.oceaneng.2012.12.030.

3. Politis CG, Papagiannopoulos A, Belibassakis KA, Kaklis PD, Kostas K V., Ginnis AI, et al. An isogeometric BEM for exterior potential-flow problems around lifting bodies. 11th World Congr. Comput. Mech. WCCM 2014, 5th Eur. Conf. Comput. Mech. ECCM 2014 6th Eur. Conf. Comput. Fluid Dyn. ECFD 2014, 2014, p. 2433-44.

4. Jasak H, Vukčević V, Gatin I, Lalović I. CFD validation and grid sensitivity studies of full scale ship self propulsion. Int J Nav Archit Ocean Eng 2019;11:33-43. doi:10.1016/j.ijnaoe.2017.12.004.

5. Khan H, Ahsan A, Chauhdhry KA. Review of Modern Trend for Numerical Model Testing in Worldwide Towing Tanks. Proc. 2019 16th Int. Bhurban Conf. Appl. Sci. Technol. IBCAST 2019, 2019, p. 732-43. doi:10.1109/IBCAST.2019.8667239.

6. Piegl L, Tiller W. The NURBS book. Choice Rev Online 1997;35:35-0952-35-0952. doi:10.5860/ choice.35-0952.

7. Cottrell JA, Hughes TJR, Bazilevs Y. Isogeometric Analysis: Toward Integration of CAD and FEA. 2009. doi:10.1002/9780470749081.

8. Quarteroni A. Isogeometric analysis. Model. Simul. Appl., vol. 16, 2017, p. $267-92$. doi:10.1007/978-3-319-49316-9 11.

9. Rjasanow S, Steinbach O. The Fast Solution of Boundary Integral Equations. Boston, MA: Springer US; 2007. doi:10.1007/0-387-34042-4.

10. Jaswon MA. Boundary Integral Equations. Springer Berlin Heidelberg; 1984.

11. Bland JA, Pozrikidis C. Boundary Integral and Singularity Methods for Linearised Viscous Flow. vol. 77. Cambridge University Press; 1993. doi:10.2307/3619810.

12. Arthur DW, Davis PJ, Rabinowitz P. Methods of Numerical Integration. vol. 70. 1986. doi: $10.2307 / 3615859$ 
13. Telles JCF. A self-adaptive co-ordinate transformation for efficient numerical evaluation of general boundary element integrals. Int J Numer Methods Eng 1987;24:959-73. doi:10.1002/ nme.1620240509.

14. Carley M. Numerical quadratures for singular and hypersingular integrals in boundary element methods. SIAM J Sci Comput 2007;29:1207-16. doi:10.1137/060666093.

15. Bassanini P, Casciola CM, Lancia MR, Piva R. Edge singularities and kutta condition in 3D aerodynamics. Meccanica 1999;34:199-229. doi:10.1023/A:1004571915758.

16. Wang C, Zou ZJ. A 3D panel method for hydrodynamic forces on hydrofoils. Chuan Bo Li Xue/ Journal Sh Mech 2001;5:19-25.

17. Drela M. XFOIL: an analysis and design system for low Reynolds number airfoils. Low Reynolds Number Aerodyn. Proc. Conf., Notre Dame, U.S.a., June 5-7, 1989 \}Edited By T.J. Mueller]. (Lecture Notes, Springer; 1989, p. 1-12. doi:10.1007/978-3-642-84010-4_1. 
